\title{
Simultaneous Recruitment of Drug Users and Men Who Have Sex with Men in the United States and Russia Using Respondent-Driven Sampling: Sampling Methods and Implications
}

\author{
Martin Y. Iguchi, Allison J. Ober, Sandra H. Berry, Terry Fain, \\ Douglas D. Heckathorn, Pamina M. Gorbach, Robert Heimer, \\ Andrei Kozlov, Lawrence J. Ouellet, Steven Shoptaw, and William A. Zule
}

\begin{abstract}
The Sexual Acquisition and Transmission of HIV Cooperative Agreement Program (SATHCAP) examined the role of drug use in the sexual transmission of the human immunodeficiency virus (HIV) from traditional high-risk groups, such as men who have sex with men (MSM) and drug users (DU), to lower risk groups in three US cities and in St. Petersburg, Russia. SATHCAP employed respondent-driven sampling (RDS) and a dual high-risk group sampling approach that relied on peer recruitment for a combined, overlapping sample of MSM and DU. The goal of the sampling approach was to recruit an RDS sample of MSM, DU, and individuals who were both MSM and $D U(M S M / D U)$, as well as a sample of sex partners of MSM, DU, and MSM/DU and sex partners of sex partners. The approach efficiently yielded a sample of 8,355 participants, including sex partners, across all four sites. At the US sites-Los Angeles, Chicago, and Raleigh-Durham-the sample consisted of older (mean age $=41$ years), primarily black MSM and DU (both injecting and non-injecting); in St. Petersburg, the sample consisted of primarily younger (mean age $=28$ years) MSM and DU (injecting). The US sites recruited a large proportion of men who have sex with men and with women, an important group with high potential for establishing a generalized HIV epidemic involving women. The advantage of using the dual high-risk group approach and RDS was, for the most part, the large, efficiently recruited samples of MSM, DU, and MSM/DU. The disadvantages were a recruitment bias by racelethnicity and income status (at the US sites) and under-enrollment of MSM samples because of short recruitment chains (at the Russian site).
\end{abstract}

KEYWORDS Respondent-driven sampling, HIV, MSM, MSMW, DU, IDU, SATHCAP

\section{INTRODUCTION}

The potential spread of the human immunodeficiency virus (HIV) from its concentration in particular groups to the general population is a growing concern

Iguchi, Ober, Berry, and Fain are with the RAND Corporation, Santa Monica, CA, USA; Iguchi and Gorbach are with the School of Public Health, University of California, Los Angeles (UCLA), Los Angeles, CA, USA; Heckathorn is with the Cornell University, Ithaca, NY, USA; Heimer is with the Yale University, New Haven, CT, USA; Kozlov is with the Biomedical Center, St. Petersburg, Russia; Ouellet is with the School of Public Health, University of Illinois at Chicago (UIC), Chicago, IL, USA; Shoptaw is with the David Geffen School of Medicine, University of California, Los Angeles (UCLA), Los Angeles, CA, USA; Zule is with the Research Triangle Institute (RTI) International, Research Triangle Park, NC, USA.

Correspondence: Martin Y. Iguchi, PhD, RAND Corporation, 1776 Main Street, Santa Monica, CA 90407, USA. (E-mail: iguchi@rand.org) 
in both the USA and Russia. Of particular concern is HIV transmission from traditional higher risk groups, such as drug users (DU) and men who have sex with men (MSM), to lower risk groups, such as non-drug-using sexual partners, through sexual intercourse. Sexual links between higher risk groups and lower risk groups are of special concern not only because they contribute to the spread of HIV but also because they could lead to a generalized epidemic. ${ }^{1}$ A growing body of literature suggests that drug use may be influencing sexual behaviors that increase HIV transmission, ${ }^{2-6}$ although little is known about the role of drug use in sexual transmission from high- to lower risk groups. This paper describes the methods and sampling results of the National Institute on Drug Abuse (NIDA) Sexual Acquisition and Transmission of HIV Cooperative Agreement Program (SATHCAP), a multi-site study designed to assess the role of drugs in the sexual transmission of HIV among higher risk groups and from high- to lower risk groups in the USA and Russia.

Between 40,000 and 56,000 new cases of HIV are diagnosed each year in the USA, ${ }^{7,8}$ and heterosexual sex is the mode of HIV exposure that has increased the most rapidly. ${ }^{9}$ In $2005,49 \%$ of all new cases in the USA were among men who reported having sex with another man, $17 \%$ were among men and women who reported injection drug use, and $32 \%$ were among men and women who reported heterosexual intercourse. ${ }^{8}$ Despite the relatively low and slow incidence of HIV among heterosexuals in the USA compared with that in many developing countries, the significant ongoing sexual transmission of HIV among MSM and the recent increases in cases among heterosexuals are a cause for concern. In 2006, an estimated 39,400 persons were diagnosed with HIV in 22 US states, and classification using the BED assay showed that $31 \%$ of these infections were recent. These data suggest that the number of new infections for the USA in 2006 was 56,300 , with an estimated incidence rate of 22.8 per 100,000 population. ${ }^{10}$

While the USA is seeing an increase in HIV transmission through sexual intercourse, the Russian Federation is experiencing a rapidly growing epidemic of HIV transmission primarily through injection drug use (IDU). In each of the past 5 years, 30,000 to 50,000 new infections have been diagnosed, at least two thirds of which were transmitted through injection drug use. ${ }^{11}$ However, there is growing evidence of increased heterosexual transmission ${ }^{11}$ and of sexual transmission from IDU to non-IDU. ${ }^{12}$ The epidemic patterns are vastly different in the USA and Russia, but they share common trajectories of HIV transmission from higher risk groups to lower risk groups such as non-drug users.

NIDA's SATHCAP brought together researchers from the University of California, Los Angeles (UCLA); the University of Chicago at Illinois (UIC); Research Triangle Institute International in Raleigh-Durham (RTI), North Carolina; Yale University, with the Biomedical Center (Yale/BMC) in St. Petersburg, Russia; and the RAND Corporation. The key research questions for SATHCAP were: (1) To what extent do HIV infections among DU and MSM populations spread to uninfected non-DU and non-MSM individuals through sexual activity? (2) What is the role of drugs in this spread? (3) What individual, behavioral, network, and structural characteristics determine the speed, extent, and path of this spread?

To answer these questions, SATHCAP conducted a cross-sectional study across four sites using a consistent sampling method, a common questionnaire, and similar biological testing. Each site used respondent-driven sampling (RDS) to recruit MSM and DU. Participants recruited MSM and DU using the RDS method and then recruited their sexual partners. MSM and DU and their sexual partners reported individual-level, partner-level, and event-level drug using and sexual risk behaviors 
and their determinants so that the rate, speed, and extent of sexual transmission to the general population could be estimated. This paper provides background information on SATHCAP's research aims and describes the study's focus, its design, and the RDS sampling approach.

\section{BACKGROUND}

\section{The Role of Drugs in the Sexual Transmission of HIV}

Several recent studies have suggested that drugs-methamphetamine and crack cocaine in particular-are contributing to increased sexual risk behaviors and increased HIV transmission in the USA and abroad. Among MSM in the USA, studies have shown a consistent correlation between methamphetamine use and high-risk sexual behaviors ${ }^{13,14}$ and between methamphetamine use and HIV incidence. ${ }^{4,15}$ Increased risk of HIV through sexual transmission among both men and women in the USA also is associated with smoking crack cocaine. ${ }^{16-20}$ Crack smokers demonstrate an even higher rate of risky sexual behavior than do cocaine injectors. ${ }^{3}$ Related patterns are evident in places outside the USA. In Russia, where methamphetamine-related drugs are the most common stimulant and injection heroin is the most common type of drug, studies suggest that unprotected heterosexual sex between IDU and non-IDU sexual partners may be contributing to the spread of HIV to the larger population. ${ }^{21}$ Evidence connecting stimulants to high-risk sexual behavior continues to mount, but little is currently known about the social epidemiology of the sexual transmission of HIV from higher risk groups, such as MSM and IDU, to lower risk groups, such as non-drug-using women and heterosexual men, and about the role of drugs in this process.

\section{The Use of RDS to Recruit Two "Hidden" Populations}

Traditional Methods for Recruiting Hidden Populations "Hidden" populations are those for which no sampling frame exists and for which there are usually privacy concerns because of stigmatized or illicit behavior. In the context of HIV surveillance and behavioral research, both DU and MSM are considered hidden populations and thus are difficult to study. ${ }^{22,23}$ Many of these individuals, particularly those with no fixed address, are not easily reached through household surveys or venue-based sampling. Many do not present for health care until they become extremely ill. Because traditional probability sampling can be quite costly and can often miss many marginalized and hidden individuals, HIV researchers often rely on nonprobability methods to recruit hidden populations and to encourage study participation. ${ }^{24,25}$ Among the more common non-probability sampling methods are snowball sampling and other chain referral methods, targeted sampling, key informant sampling, and venue-based sampling, as described by Magnani et al. ${ }^{24}$ However, non-probability sampling methods contain biases of unknown magnitude and direction. ${ }^{24,25}$

Respondent-Driven Sampling Several recent studies have demonstrated that RDS can efficiently yield large samples of both IDU and DU ${ }^{22,26-29}$ as well as MSM. ${ }^{30-34}$ RDS is a peer-driven chain referral method that relies on a structured system of recruitment procedures and financial incentives to encourage recruitment and participation. RDS also employs post-stratification weighting procedures. Together, these characteristics are thought to reduce biases typically associated with non- 
random recruitment. ${ }^{22,23,25}$ Whereas non-RDS methods likely oversample larger personal networks, venue attendees, and DU with more visible street lives, ${ }^{35}$ RDS is designed to avoid, reduce, and control for this pitfall.

RDS recruitment begins with a small number of non-randomly selected "seeds." Seeds used in RDS generally are high-energy, well-connected members of a network. The reason to select seeds with these characteristics is to jump-start the recruitment process and reduce bias by speeding the attainment of equilibrium. ${ }^{24,25}$ Seeds are given incentives for successful recruitment of people they know who qualify for the study. ${ }^{25}$ Recruitment then expands through multiple recruitment waves, with each wave's recruits becoming recruiters and receiving compensation for recruiting eligible peers. In order to prevent oversampling from larger personal networks, RDS limits the number of individuals that may be recruited by a single recruiter, thus allowing for an increase in social distance between seeds, their recruits, and later waves. Theoretically, RDS samples reach an "equilibrium" and stabilize after multiple waves. ${ }^{22}$ Equilibrium in RDS refers to the point at which sample characteristics cease to fluctuate and, theoretically, approximate characteristics of participants' networks. ${ }^{25}$ An RDS sample is only self-weighting if two conditions are met: (1) groups of analytic interest (such as males and females, HIV positives and negatives, or racial/ethnic groups) have equivalent network sizes, and (2) groups recruit with equal efficiency. ${ }^{36}$ Because these two conditions typically are not met, ${ }^{36}$ the overall sample composition frequently does not correspond to population estimates derived from the RDS method. In these cases, post-stratification weights are required to yield population estimates that control for these sources of bias. The theoretical and mathematical foundations and procedures of RDS are described in detail elsewhere. ${ }^{23,25}$

Despite some noteworthy limitations, most studies conclude that RDS is an efficient (i.e., effectiveness and speed at recruiting target populations) way to recruit large samples of DU (both injecting and non-injecting) ${ }^{22,26-29}$ and MSM $^{30-32,34}$ both within and outside the USA. Most studies conclude that the demographics of samples recruited through RDS reflect the general demographics of the IDU and DU in similar study locations, though some suggest that recruitment efficiency may vary by population density, availability of public transportation, and the ability to recruit productive seeds. Other studies note that RDS samples may not be representative of target populations. ${ }^{26,29}$

RDS is Efficient in Recruiting IDU and DU In a pilot study in New York City designed to assess the effectiveness of RDS in recruiting IDU and DU, Abdul-Quadar et al. ${ }^{24}$ enrolled 618 IDU and DU from eight seeds over a period of 13 weeks, with $43 \%$ of the sample having injected in the previous 6 months, $19 \%$ having injected prior to the previous 6 months, and 38\% never having injected drugs but having sniffed or smoked a drug. Robinson et al., ${ }^{37}$ who conducted a pilot to compare the effectiveness of RDS and targeted sampling in three US cities, also found RDS to be efficient. In 8 weeks, they recruited 102 IDU from three seeds in Detroit, 48 IDU from one seed in Houston, and 118 IDU from two seeds in New Orleans. Wang et al. ${ }^{28}$ demonstrated that RDS can be effective for recruiting non-injecting DU, specifically MDMA/ecstasy users and illicit stimulant users ${ }^{29}$ in non-metropolitan settings in central Ohio. They were able to recruit 374 active MDMA/ecstasy users from 28 initial seeds and 249 stimulant users from 11 seeds. Recruitment was slow, however, and both of these studies reported a high number of unproductive seeds. Grau et al. $^{38}$ efficiently recruited 237 opioid abusers in Cumberland County 
(Portland), Maine, in 10 weeks from 21 seeds. One seed yielded 17 waves of recruitment and over half (120) of the study participants. However, recruitment was concentrated in the inner city, and chains of recruits infrequently reached into the suburban towns of Cumberland County. Stormer at al. ${ }^{27}$ found RDS to be an efficient way to recruit IDU in two cities in two different countries-Tirana, Albania and St. Petersburg, Russia-successfully recruiting 200 IDU from each site within 8 weeks. In the same amount of time, Frost et al. ${ }^{26}$ recruited 200 IDUs from each of two border cities in Mexico-Ciudad Juarez and Tijuana.

RDS is Efficient in Recruiting MSM Ramirez-Valles et al. ${ }^{30}$ demonstrated that RDS is an appropriate and efficient way to recruit a hidden population of gay, bisexual, and transgender (GBT) men in urban areas. Their study began with seven seeds in Chicago and nine in San Francisco and reached a target sample of 100 GBT Hispanic men at each site in 7 weeks in Chicago and 13 weeks in San Francisco. Johnston et al. ${ }^{31}$ also demonstrated that RDS can be effective in recruiting MSM. Beginning with eight seeds, they recruited 523 MSM in Dhaka, Bangladesh in 11 weeks. In a study of circumcision status among MSM, Millet et al. ${ }^{32}$ used RDS to recruit 1,079 black MSM and 957 Hispanic MSM from New York City, Los Angeles, and Philadelphia. Deiss et al., ${ }^{34}$ reporting on the same study as Frost et al. ${ }^{26}$ found that $47 \%$ of all male IDU recruited in Tijuana and $13 \%$ of all male IDU recruited in Ciudad Juarez were MSM/IDU, suggesting that RDS recruitment might be effective in recruiting dual high-risk populations.

As with All Sampling Approaches, RDS Has Limitations Although RDS has several strengths and may reflect estimated population compositions, as with all populationbased studies, it is not immune from sampling bias. ${ }^{26,29}$ Post-stratification weighting is proposed as a way to correct for sampling bias in RDS samples, but some researchers suggest that it cannot fully account for bias introduced by non-random selection from personal networks. ${ }^{29,39}$ Frost et al. ${ }^{26}$ note that the post-stratification weighting process depends on subject reports of network size, and that despite the RDS method's assumption that respondents can accurately report their network sizes, there may be variations because of how questions about network size are asked. In addition, recall of social networks can be inaccurate and incomplete due to forgetting, and forgetting tends to increase as network size increases. ${ }^{40-42} \mathrm{Scott}^{43}$ suggests potential methodological and ethical violations of RDS, but multiple researchers criticize Scott's own methods ${ }^{44-46}$ and point out that violations suggested by Scott are exaggerated and not unique to RDS sampling. ${ }^{44,45}$ Thus, while RDS is an efficient means of recruiting large samples of hidden populations, inaccuracies in estimates of network sizes may lead to biases. Limitations notwithstanding, most studies conclude that RDS is an efficient and cost-effective way to recruit large hidden populations of IDU, DU, and MSM and suggest that RDS, in the right circumstances, may be a useful way to recruit samples that represent specific social networks and even the underlying hidden population from which the sample is drawn.

\section{Recruitment of the Dual High-Risk Group}

Because of the reported effectiveness of RDS in recruiting IDU, DU, and MSM, SATHCAP employed the basic concepts and procedures of RDS in a new way, designed to simultaneously recruit a large, dual higher risk group composed of drug 
users (injecting and non-injecting) and MSM, regardless of drug use, and then to recruit sex partners of members of the dual higher risk group. Recruitment of networks of drug users, MSM, and their sex partners has been conducted in social network studies using other methodologies, ${ }^{47,48}$ but no prior studies have used RDS to recruit a dual risk group and few, if any, have specifically targeted two relatively separate risk groups, i.e., drug users (injecting or non-injecting) who may or may not be MSM and MSM who may or may not be drug users and their male or female sexual partners. The goal was to form a large base of individuals at high risk for HIV whose interrelationships could be studied and to identify potential bridging sex partners who might transmit HIV beyond the dual higher risk group to those at lower risk.

\section{METHODS}

\section{Study Design}

SATHCAP was a cross-sectional study conducted in two phases. For the three US sites, phase 1 recruitment took place between September 2005 and December 2006 and phase 2 between November 2006 and August 2008. In Russia, phase 1 recruitment took place between November 2005 and December 2006 and phase 2 between February 2007 and August 2008. The two phases were essentially identical except for slight changes to the recruitment scheme in phase 2 to adjust for underrecruitment of sex partners in phase 1 . Phase 1 respondents were not eligible to participate in phase 2 . In the analyses presented herein, we combine the results from the two phases.

\section{Seed Selection}

All sites began recruitment in each phase with the selection of seeds that met study eligibility criteria and the requirements of RDS-networks of substantial size and a willingness to recruit. The UCLA Office for the Protection of Research Subjects allowed only passive recruitment of seeds through flyers and advertisements (i.e., they could not approach potential seeds but had to wait for seeds to contact them); the other sites allowed active recruitment in which study staff could identify and approach community members who they though met eligibility criteria and invite them to participate. Each site began the study with a small number of seeds and then added seeds as needed throughout the study to boost recruitment when a recruitment chain ended or to boost recruitment in one of the higher risk groups (MSM, DU/IDU). Table 1 illustrates the composition of all seeds selected throughout both phases of the study.

\section{Eligibility Screening}

Each site stored eligibility information in a record management system (RMS). The RMS included an eligibility screening system, descriptive characteristics of participants, confirmation of consent, status of questionnaire and biological testing completion, and the numbers and dates of coupons distributed by each participant. All potential participants first were screened to determine whether their coupons were valid. Next, participants answered a series of questions to determine whether they met eligibility criteria for the type of coupon they wished to redeem. For example, a participant with a DU/IDU/MSM coupon had to report using 
TABLE 1 Seed composition

\begin{tabular}{lcccc}
\hline & RTI $(n=96)(\%)$ & UCLA $(n=120)(\%)$ & UIC $(n=47)(\%)$ & Yale/BMC $(n=156)(\%)$ \\
\hline Male & 75 & 94 & 64 & 85 \\
White & 42 & 28 & 36 & 100 \\
Black & 56 & 31 & 30 & \\
Hispanic & 2 & 35 & 30 & \\
Other & & 7 & 4 & 55 \\
Non-IDU/DU MSM & 15 & 31 & & 36 \\
IDU/DU & 65 & 22 & 74 & 9 \\
>MSM+IDU/DU & 20 & 47 & 26 & 23 \\
$<30$ & 19 & 15 & 32 & 4 \\
$30-39$ & 22 & 30 & 32 & 2 \\
$40-49$ & 44 & 38 & 23 & \\
$50+$ & 16 & 17 & 13 & \\
\hline & & & & \\
\hline
\end{tabular}

methamphetamine, heroin, crack cocaine, or powder cocaine in the previous 6 months, or injecting some other drug in the previous 6 months, and/or having anal sex with another man in the previous 6 months. Participants redeeming sex partner coupons had to report having had sex in the previous 6 months with the person who recruited them into the study. For those claiming MSM status only, the RMS screened to confirm that the recruiter was a male. The RMS also screened for duplicate participants by comparing new participants' demographic characteristics (such as birth date and race/ethnicity), biometric measurements of arm length and wrist circumference, and physical features (such as tattoos and scars) with those of existing participants. The RMS displayed lists of potential matches with which to compare new recruits and eliminate duplicates.

\section{Interviews and Biological Sampling}

All eligible participants were asked to complete three questionnaires and to provide biological samples for HIV and other sexually transmitted disease testing. All questionnaires were self-administered computerized interviews (computer-assisted self-interview) with audio (in Chicago, Los Angeles, and Raleigh-Durham) or without (in St. Petersburg).

RDS 1 Questionnaire The first questionnaire was an RDS sampling questionnaire used to collect data on social network size and composition for post-stratification assignment of RDS weights and to examine possible recruitment bias.

SATHCAP Main Questionnaire The SATHCAP Main Questionnaire (SMQ) asked participants about drug using and sexual risk behaviors, about sexual partnerships and the nature of these partnerships, and about the location of their risk activities. SMQ items were drawn from a variety of existing instruments, including the NIDA Risk Behavior Assessment, ${ }^{49,50}$ the CDC Project Mix Sexual Behavior Assessment, ${ }^{51}$ the EXPLORE Study Instrument, ${ }^{52}$ the CDC YMS Study Instrument, ${ }^{53}$ and the HIV Partnership Survey. ${ }^{54}$ Injection and sexual risk behavior questions were asked at the global level (i.e., whether participants had ever or recently engaged in a certain activity) and at the event level (i.e., whether they had engaged in certain behaviors with specific recent partners whose initials they provided at the start of the injection 
and sexual risk sections of the questionnaire). In the injection risk section, participants could report on up to four partners with whom they had injected drugs in the previous 6 months. These included three recent drug injection partners and the primary drug-injecting partner if he or she was not one of the three. In the sexual risk behavior section, participants could report on up to six partners with whom they had had sex in the previous 6 months. These included three recent partners, as well as an IDU partner who was also a sexual partner, their main sexual partner, and a female sexual partner of an MSM, if these partners were not among the initial three. In phase 2 of the study, participants also could answer questions about their recruiter if the recruiter was not among the initial three drug injection or sexual partners mentioned. The same questionnaire was administered at each site. The SMQ reflected the overall study design by emphasizing the identification of individuals serving a functional role as bridges for HIV transmission from identified higher risk groups to lower risk groups via sexual transmission.

RDS 2 Questionnaire An RDS questionnaire designed to assess the characteristics of individuals who declined coupons was administered to participants returning to obtain payment for successful referrals. In this third questionnaire, participants were queried about individuals to whom they had given coupons but who had not accepted or redeemed them.

Biological Testing This questionnaire also was designed to facilitate the examination of recruitment bias. All sites tested participants for HIV, syphilis, gonorrhea, and Chlamydia trachomatis. In addition, the Los Angeles site tested MSM for anal human papilloma virus, Raleigh-Durham tested all participants for hepatitis $\mathrm{C}$, and St. Petersburg tested all participants for hepatitis B and C. The number of tests conducted by all sites was limited due to cost considerations.

\section{Multi-Level Recruitment: Dual Higher Risk Group to Sex Partner to Sex Partner of Sex Partner}

Upon completing the RDS 1 and the SMQ questionnaires and biological specimen collection, participants who completed all parts of the study were eligible to distribute coupons. Coupons were dollar-bill-sized colored paper that contained a coupon number for later eligibility screening as well as contact information for the study. A map of the study location was on the back of the coupons. The types and colors of coupons distributed by study participants differed by risk group classification.

In phase 1 of the study, dual higher risk group participants were given coupons to give to three people who also met the dual higher risk group definition: "any person who used heroin, methamphetamine, cocaine, or crack, or injected another drug in the past six months," OR "any man who had anal sex with another man in the past six months." Dual higher risk group participants were also given two "sex partner" coupons with which to recruit people who met study criteria for a sex partner: "any person with whom you have had sex in the past six months." In addition, participants were given one "special interest sex partner" coupon. Those participants who were IDU/DU were to use this coupon to recruit a non-drug-using sex partner with whom they had had sex in the previous 6 months, and participants who were MSM were to use it to recruit women with whom they had had sex in the previous 6 months. Sex partners also were eligible to become recruiters. The sex 
partners of dual higher risk group members were given three sex partner coupons with which to recruit anyone with whom they had had sex in the previous 6 months. Sex partners of sex partners could not become recruiters and thus did not distribute coupons.

Study investigators altered recruitment in phase 2 to simplify coupon distribution for recruiters and to adjust for low recruitment of non-drug-using sex partners in phase 1 . In phase 2 , dual higher risk group participants initially received two coupons to give to two people who met the definition of the dual higher risk group (MSM or IDU/DU). They also received two "opposite sex partner" coupons with which to recruit female sex partners if they were male and male sex partners if they were female. Participants who redeemed opposite sex partner coupons and met the sex partner criteria were given two opposite sex partner coupons with which to recruit up to two of their own-sex partners. All sites increased the number of dual higher risk group coupons to four by the middle of the phase 2 recruitment period to increase recruitment of dual higher risk group participants.

All participants, including seeds, were compensated for their time and participation in the study and for every eligible person they recruited (i.e., provided coupons to) who was willing to be screened. Payments for participation in the research protocol and recruiter compensation differed by site due to variations in local practices and Institutional Review Board requirements.

\section{Eligibility Verification at the Analysis Stage}

Although initial eligibility screenings were conducted carefully and consistently at all sites, participants may have had prior knowledge about eligibility criteria and could have provided false responses to the eligibility screening questions in order to obtain the benefits of study participation. Therefore, we applied a secondary algorithm to verify eligibility for inclusion in the analysis. For dual higher risk group membership (MSM or IDU/DU), individuals (1) had to have been recruited by another dual higher risk group member and (2) had to have answered affirmatively to questions during the main interview indicating IDU/DU or MSM status. To be eligible as a sex partner, respondents had to have reported during the interview that they had had sex with the person who gave them a coupon. Participants who were found to be inconsistent on these criteria were excluded from analysis. Although secondary screening also was based on self-report, the results had no impact on participant eligibility for participant payments, so participants did not have an ongoing incentive to provide false answers.

Requiring consistent responses between the questionnaire and the eligibility screen for analytic purposes provided additional assurance of accurate risk group classification. However, as in many studies using self-report for eligibility, there was no guarantee that our additional screening identified all who did not meet the criteria. The total number of participants across all sites that were excluded from analysis in phase 1 because they were found to be an ineligible MSM or IDU/DU or a sexual partner recruited by an ineligible MSM or IDU/DU was $123(3.8 \%)$ of 3,268 participants. The total number excluded in phase 2 was $242(4.8 \%)$ of 5,087 participants.

In addition, while a recruit's coupon provided an initial risk classification, further examination at the data analysis stage showed that participants frequently belonged to more than one risk group. For example, participants coming in as sex partners and as sex partners of sex partners frequently met criteria for the primary risk group categories (MSM or IDU/DU). Because the primary purpose of the study 
was to determine the spread of sexually transmitted HIV from higher risk groups to lower risk groups, sex partners found to meet criteria for the primary risk group were assigned to the dual higher risk group for analytic purposes. A designation of "sex partner only" was made for sex partners who were not also members of either primary risk group.

\section{Assessment of Recruitment Bias and Network Representation}

To evaluate potential recruitment bias at each site, we compared the overall composition of the samples recruited at each site with the reported composition of those who refused coupons to determine non-participation bias. Next, we evaluated the RDS reciprocity assumption to determine if recruiters recruited people known to them. We also examined recruitment homophily (in-group recruitment), network equilibrium, and non-response bias to determine their relative effects on the final composition of the RDS sample, and we tested the RDS random recruitment assumption, that is, whether participants recruited randomly from their personal networks. Finally, to determine the level of bias created by recruitment of like individuals, we assessed sample equilibrium to determine whether the samples eventually reached a point of stabilization.

\section{RESULTS}

\section{Recruitment}

The sites spent between 12 and 14 months recruiting during phase 1 of the study and up to 21 months during phase 2 (see Table 2). In phase 1, the Chicago site recruited the most participants $(n=1,068)$ from the fewest number of seeds $(n=5)$. Raleigh-Durham and Los Angeles recruited fewer participants $(n=881$ and $n=797$, respectively) from a larger number of seeds $(n=17$ and $n=25$, respectively). St. Petersburg required the greatest number of seeds $(n=48)$ because of short recruitment chains and somewhat less efficient recruitment (i.e., fewer participants, slower recruitment) than was the case at the US sites, resulting in the enrollment of fewer participants $(n=522)$. Phase 2 extended longer than phase 1 in an effort to maximize the length of recruitment chains. All sites employed a substantially higher

TABLE 2 Summary of recruitment for study phases 1 and 2

\begin{tabular}{lccrc}
\hline & Raleigh-Durham & Los Angeles & Chicago & St. Petersburg \\
\hline Phase 1 & & & & \\
Seeds & 17 & 25 & 5 & 48 \\
Months of recruitment & 12 & 13 & 14 & 13 \\
Primary risk group recruited & 536 & 426 & 757 & 346 \\
Sex partners recruited & 345 & 371 & 311 & 176 \\
Total recruited phase 1 & 881 & 797 & 1,068 & 522 \\
Phase 2 & & & & \\
Seeds & 79 & 95 & 42 & 108 \\
Months of recruitment & 19 & 17 & 21 & 18 \\
Primary risk group recruited & 664 & 665 & 1,688 & 343 \\
Sex partners recruited & 440 & 180 & 1,051 & 56 \\
Total recruited Phase 2 & 1,104 & 845 & 2,739 & 399 \\
\hline
\end{tabular}


TABLE 3 Percentage of participants recruited from dual high-risk group (MSM or IDU/DU) coupons (behaviorally defined)

\begin{tabular}{lcccc}
\hline & $\begin{array}{l}\text { Raleigh-Durham } \\
(n=1,200)(\%)\end{array}$ & $\begin{array}{l}\text { Los Angeles } \\
(n=1,091)(\%)\end{array}$ & $\begin{array}{l}\text { Chicago } \\
(n=2,445)(\%)\end{array}$ & $\begin{array}{l}\text { St. Petersburg } \\
(n=689)(\%)\end{array}$ \\
\hline IDU & & & & \\
Male & 14 & 14 & 22 & 55 \\
Female & 5 & 4 & 11 & 13 \\
IDU/MSM & 1 & 9 & 1 & 0 \\
IDU/MSM/W & 5 & 10 & 4 & 1 \\
DU & & & & 2 \\
Male & 34 & 17 & 32 & 1 \\
Female & 28 & 4 & 19 & 1 \\
DU/MSM & 4 & 18 & 4 & 21 \\
DU/MSM/W & 4 & 14 & 6 & 4 \\
MSM & 3 & 8 & 1 & 100 \\
MSM/W & 1 & 3 & 0 & \\
Total & 99 & 101 & 100 & \\
\hline
\end{tabular}

Percentages do not total $100 \%$ due to rounding

number of seeds in phase 2 and, with the exception of the St. Petersburg site, recruited more participants than they had in phase 1 .

\section{Risk Group Composition}

In both phases of the study, IDU/DU who were not MSM made up the majority of participants recruited via dual higher risk group (MSM or IDU/DU) coupons in Raleigh-Durham, Chicago and St. Petersburg (see Tables 3 and 4). In Los Angeles, about half of the participants recruited via dual higher risk group coupons in both phases of the study were MSM or men who have sex with men and with women

TABLE 4 Percentage of participants recruited from sex partner coupons (behaviorally defined)

\begin{tabular}{|c|c|c|c|c|}
\hline & $\begin{array}{l}\text { Raleigh-Durham } \\
(n=785)(\%)\end{array}$ & $\begin{array}{l}\text { Los Angeles } \\
(n=551)(\%)\end{array}$ & $\begin{array}{l}\text { Chicago } \\
(n=1,362)(\%)\end{array}$ & $\begin{array}{l}\text { St. Petersburg } \\
(n=232)(\%)\end{array}$ \\
\hline \multicolumn{5}{|l|}{ IDU } \\
\hline Male & 6 & 5 & 9 & 25 \\
\hline Female & 8 & 10 & 11 & 34 \\
\hline IDU/MSM & 1 & 6 & 0 & \\
\hline IDU/MSM/W & 4 & 12 & 3 & \\
\hline \multicolumn{5}{|l|}{ DU } \\
\hline Male & 23 & 14 & 24 & 1 \\
\hline Female & 24 & 19 & 35 & 3 \\
\hline DUMSM & 2 & 8 & 1 & 0 \\
\hline DUMSM/W & 4 & 12 & 5 & 0 \\
\hline MSM & 1 & 3 & 0 & 7 \\
\hline $\mathrm{MSM} / \mathrm{W}$ & 1 & 1 & 0 & 1 \\
\hline \multicolumn{5}{|c|}{ Sex partner only } \\
\hline Male & 11 & 4 & 5 & 6 \\
\hline Female & 16 & 6 & 7 & 21 \\
\hline Total & 101 & 100 & 100 & 98 \\
\hline
\end{tabular}

Percentages do not total $100 \%$ due to rounding 
(MSMW) IDU/DU. High proportions of MSM or IDU/DU coupon holders at all three US sites identified themselves as MSMW DU in both phases of the study. Note that risk groups in Tables 3 and 4 are defined by behaviors reported in the SMQ rather than by coupon type.

\section{Sample Characteristics}

Tables 5 and 6 give the demographics for the two sets of coupon recruits, respectively, for both phases of the study. As shown, the mean age for both dual higher risk group (MSM or IDU/DU) and sex partner coupon recruits was around 40 years at each US site and 28 years at the St. Petersburg site. All samples were predominantly male, with a small increase in the proportion of females in phase 2 , consistent with the focus on recruiting partners of the opposite sex. In both phases, the majority of participants in each of the US samples were black, with modest proportions of Hispanic participants at the Los Angeles and Chicago sites. Being employed, either full or part-time, among dual higher-risk group coupon holders ranged from $13 \%$ at the Chicago site to $44 \%$ in St. Petersburg, with a similar range among sex partner coupon holders. High proportions of the sample at each site reported being homeless at some time during the previous year, with a range among dual higher risk group coupon holders of $20 \%$ (St. Petersburg) to 56\% (Los Angeles).

\section{Assessment of Recruitment Bias}

To better understand the sources and relative contributions of recruitment bias, we examined discrepancies between network compositions of those recruited and those who refused coupons; we explored the relationship between recruiters and recruits to determine whether recruits were known to recruiters, an important requirement of RDS; we assessed the samples for possible homophily bias; we conducted a

TABLE 5 Demographics of dual high-risk group (MSM/IDU/DU) coupon recruits

\begin{tabular}{lcccr}
\hline & $\begin{array}{l}\text { Raleigh-Durham } \\
(n=1,200)\end{array}$ & $\begin{array}{l}\text { Los Angeles } \\
(n=1,091)\end{array}$ & $\begin{array}{l}\text { Chicago } \\
(n=2,445)\end{array}$ & $\begin{array}{l}\text { St. Petersburg } \\
(n=689)\end{array}$ \\
\hline Mean age & 40 & 43 & 44 & 28 \\
Male (\%) & 66 & 92 & 70 & 87 \\
Ethnicity & & & & \\
Black (\%) & 75 & 50 & 73 & 100 \\
White (\%) & 21 & 22 & 9 & \\
Hispanic (\%) & 2 & 23 & 16 & \\
Other (\%) & 2 & 5 & 1 & 32 \\
Employment & & & & \\
Disabled (\%) & 24 & 42 & 28 & \\
Unemployed (\%) & 48 & 37 & 56 & \\
Full-time (\%) & 15 & 7 & 5 & \\
Part-time (\%) & 10 & 9 & 8 & \\
Income & & & & \\
Mean & & 63 & 72 & \\
$0-\$ 500(\%)$ & 69 & 21 & 18 & \\
\$501-\$1,000 (\%) & 18 & 56 & 41 & \\
Homeless (\%) & 46 & & & \\
\hline
\end{tabular}


TABLE 6 Demographics of sex partner coupon recruits

\begin{tabular}{lcccr}
\hline & $\begin{array}{l}\text { Raleigh-Durham } \\
(n=785)\end{array}$ & $\begin{array}{l}\text { Los Angeles } \\
(n=551)\end{array}$ & $\begin{array}{l}\text { Chicago } \\
(n=1,362)\end{array}$ & $\begin{array}{l}\text { St. Petersburg } \\
(n=232)\end{array}$ \\
\hline Mean age & 38 & 42 & 44 & 28 \\
Male (\%) & 52 & 65 & 47 & 42 \\
Ethnicity & & & & 100 \\
White (\%) & 21 & 16 & 5 & \\
Black (\%) & 74 & 59 & 86 & \\
Hispanic (\%) & 3 & 20 & 8 & \\
Other (\%) & 2 & 4 & 1 & \\
Employment & & & & \\
Disabled (\%) & 20 & 36 & 26 & \\
Unemployed (\%) & 42 & 40 & 56 & \\
Full-time (\%) & 21 & 9 & 8 & \\
Part-time (\%) & 13 & 9 & 8 & \\
Income & & & & \\
Mean/Median & 62 & 68 & 70 & \\
$0-\$ 500$ & 16 & 19 & 20 & \\
\$501-\$1,000 & 35 & 58 & 36 & \\
Homeless & & & & \\
\hline
\end{tabular}

sensitivity analysis to test the assumption of random recruitment; and we examined points at which samples reached equilibrium.

Recruited Social Network Composition Versus Composition of Those Who Refused Coupons At the Raleigh-Durham site, racial/ethnic composition of those who refused coupons did not differ significantly (chi-square, $p<0.05$ ) from the composition of those recruited. In Los Angeles, the percentage of overall coupon refusals who were Hispanic $(21 \%)$ differed significantly from the overall percentage of recruits who were Hispanic $(18 \%) \chi^{2}(1 N=2,549)=4.04, p<0.05$. In Chicago, the percentage of overall coupon refusals who were black $(59 \%)$ differed significantly from the overall percentage of recruits who were black $(50 \%) \chi^{2}(1 N=4,841)=$ $37.03, p<0.05$, and the percentage of overall refusals who were white $(28 \%)$ differed significantly from the percentage of recruits where were white $(38 \%) \chi^{2}(1 \mathrm{~N}=$ $4,841)=48.18, p<0.05$.

Recruiter/Recruit Reciprocity An assumption of RDS is that there is a reciprocal relationship between recruiters and recruits; ${ }^{25}$ that is, recruiters are instructed to recruit people "they know" who are members of their MSM or DU networks. To test this assumption, we asked recruits how they would describe their relationship with their recruiter-friend, acquaintance, or stranger. Most participants at each site described their recruiter as a friend or acquaintance (Table 7). There were, however, a small percentage of participants at each site who said their recruiter was a stranger. This indicates some breakdown in study procedures and, in these cases, a violation of the reciprocity assumption.

Homophily The homophily index provides information about the tendency among study participants to recruit others with characteristics like or unlike their own. ${ }^{25}$ Random mixing produces homophily values that approximate zero. A homophily level can be said to approximate random mixing statistically if the confidence 


\section{TABLE 7 Recruiter/recruit reciprocity}

\begin{tabular}{lr}
\hline Raleigh-Durham (\%) & 62 \\
Friend & 30 \\
Acquaintance & 7 \\
Stranger & 57 \\
Los Angeles (\%) & 37 \\
Friend & 6 \\
Acquaintance & 61 \\
Stranger & 36 \\
Chicago (\%) & 3 \\
Friend & \\
Acquaintance & 45 \\
Stranger & 52 \\
St. Petersburg (\%) & 4 \\
Friend & 5 \\
Acquaintance & 5 \\
Stranger & \\
\hline
\end{tabular}

interval (CI) for homophily includes the zero point. When the question at issue is the extent to which homophily affects affiliation patterns, no hard line of demarcation defines low homophily, but a figure of 0.4 is commonly used. In that case, a strong plurality of affiliations (i.e., 60\%) are formed in a manner independent of homophily. ${ }^{36}$

Tables $8,9,10$, and 11 show the homophily scores by site for, respectively, risk group, gender, race/ethnicity, and HIV status. A score of 0 on a homophily index indicates equivalent recruitment across groups, a score of 1 indicates exclusive ingroup recruitment, and a score of -1 indicates exclusive out-group recruitment. When viewing homophily scores along the diagonal in the tables, a score of 1 indicates that individuals recruit only others like themselves; a score of -1 indicates

TABLE 8 Risk group homophily by site

\begin{tabular}{lrrr}
\hline & \multicolumn{1}{l}{ DU } & DUMSM & \multicolumn{1}{c}{ MSM } \\
\hline Raleigh-Durham & & & \\
DU & 0.443 & -0.411 & -0.588 \\
DUMSM & -0.327 & 0.306 & 0.008 \\
MSM & -0.418 & 0.089 & 0.276 \\
Los Angeles & & & \\
DU & 0.297 & -0.250 & -0.546 \\
DUMSM & -0.362 & 0.319 & -0.108 \\
MSM & -0.537 & 0.061 & 0.223 \\
Chicago & & & \\
DU & 0.460 & -0.424 & -0.784 \\
DUMSM & -0.410 & 0.384 & 0.015 \\
MSM & -0.668 & 0.335 & 0.273 \\
St. Petersburg & & & \\
DU & 0.741 & 0.004 & -0.933 \\
DUMSM & -0.544 & 0.082 & 0.455 \\
MSM & -0.965 & 0.119 & 0.836 \\
\hline
\end{tabular}


TABLE 9 Gender homophily by site

\begin{tabular}{lrr}
\hline & Male & Female \\
\hline Raleigh-Durham & & \\
Male & 0.316 & -0.316 \\
Female & -0.451 & 0.451 \\
Los Angeles & & \\
$\quad$ Male & 0.357 & -0.357 \\
Female & -0.412 & 0.412 \\
Chicago & & \\
Male & 0.377 & -0.377 \\
Female & -0.440 & 0.440 \\
St. Petersburg & & \\
Male & 0.153 & -0.153 \\
Female & -0.221 & 0.221 \\
\hline
\end{tabular}

that they recruit only others unlike themselves. Homophily index values of greater than \pm 0.7 are thought to be problematic in RDS studies because design effects are estimated at 4 or more, with steep increases in design effect as homophily values rise. $^{55}$ A homophily value of +0.7 may be interpreted as indicating that recruitment was consistent with random mixing $30 \%$ of the time and was in-group $70 \%$ of the time. Of course, increases in design effect for making point prevalence estimates across groups require an increase in sample size and more recruitment waves to overcome population segmentation. High homophily values also require an increase in recruitment waves to overcome population segmentation.

The results for the risk groups (Table 8) indicate that DU had a tendency to recruit other DU and not to recruit non-DU MSM. Similarly, non-DU MSM tended to recruit other non-DU MSM and not DU. In Chicago, however, homophily values were quite large, with DU demonstrating out-group recruitment of MSM $78 \%$ of the time and MSM demonstrating out-group recruitment of DU $67 \%$ of the time. In St. Petersburg, homophily values were even larger, with DU demonstrating in-group

TABLE 10 Race/ethnicity homophily by site

\begin{tabular}{lrrr}
\hline & Black & Hispanic & White \\
\hline Raleigh-Durham & & & \\
Black & 0.404 & -0.261 & -0.463 \\
White & -0.517 & -0.559 & 0.483 \\
Hispanic & 0.178 & -1.000 & -0.304 \\
Los Angeles & & & \\
Black & 0.486 & -0.629 & -0.355 \\
White & -0.439 & -0.156 & 0.322 \\
Hispanic & -0.560 & 0.326 & 0.037 \\
Chicago & & & \\
Black & 0.707 & -0.808 & -0.576 \\
White & -0.551 & 0.106 & 0.324 \\
Hispanic & -0.773 & 0.601 & 0.071 \\
\hline
\end{tabular}


TABLE 11 HIV status homophily by site

\begin{tabular}{lrr}
\hline & Negative & Positive \\
\hline Raleigh-Durham & & \\
HIV-negative & 0.428 & -0.428 \\
HIV-positive & -0.284 & 0.284 \\
Los Angeles & & \\
HIV-negative & 0.656 & -0.656 \\
HIV-positive & -0.630 & 0.630 \\
Chicago & & \\
HIV-negative & 0.364 & -0.364 \\
HIV-positive & -0.236 & 0.236 \\
St. Petersburg & & -0.210 \\
HIV-negative & 0.210 & 0.095 \\
HIV-positive & -0.095 & \\
\hline
\end{tabular}

recruitment of other DU 74\% of the time and out-group recruitment of MSM 93\% of the time. MSM in St. Petersburg demonstrated in-group recruitment of other MSM $84 \%$ of the time and out-group recruitment of DU $97 \%$ of the time. Of great interest is the MSM/DU group at all sites that demonstrated lower homophily values and a much greater tendency to demonstrate random mixing than either DU or MSM. These values indicate an important potential role for MSM/DU as a bridge between these two risk groups in Chicago and St. Petersburg.

A review of the homophily values for gender (Table 9) indicates that females at all of the US sites demonstrated a slight tendency to recruit other females and not to recruit males. The homophily values for males demonstrated recruitment patterns that were generally consistent with random mixing.

The racial/ethnic recruitment patterns (Table 10) are more complex. In general, black participants at the US sites demonstrated a slight tendency to recruit other blacks and not to recruit Hispanics or whites. This tendency was more pronounced in Chicago where black participants demonstrated in-group recruitment of other black participants approximately $71 \%$ of the time and out-group recruitment of Hispanic participants $81 \%$ of the time. At the same time, Hispanic participants demonstrated out-group recruitment of black participants $77 \%$ of the time. Other recruitment combinations in Chicago did not demonstrate the same level of segmentation (e.g., white participants' recruitment of Hispanic participants was consistent with random mixing almost $90 \%$ of the time).

With respect to HIV status (Table 11), in Los Angeles, HIV-negative participants tended to recruit persons who were also HIV-negative (homophily $=0.66$ ) and not to recruit persons who were HIV-positive (homophily=-0.66). HIV-positive participants responded similarly: They tended to recruit persons who were HIV-positive (homophily $=0.63$ ) and not persons who were HIV-negative $(-0.63)$.

Evaluating the Random Recruitment Assumption An analytic assumption of the RDS method holds that respondents recruit randomly from their personal networks. A means for testing this assumption involves comparing the composition of personal networks as revealed by self-reports versus actual recruitment behavior. If the assumption of random recruitment is satisfied and the self-reports are accurate, the two should vary only due to stochastic variation. 
The plausibility of this assumption depends importantly on research design. For example, the location of the interview site can be significant, as was shown in a Bridgeport, Connecticut study of IDUs. ${ }^{55}$ Sampling in an interview site located in a black neighborhood yielded only blacks, though respondents also reported knowing Hispanic IDUs. Consequently, the random recruitment assumption was violated; that is, recruitment behavior (i.e., all blacks) did not coincide with self-reported network composition (i.e., a mix of blacks and Hispanics). The solution to this problem involved moving the interview site to neutral ground, the downtown area within which no ethnic group predominated. Recruitment then yielded a mix of blacks and Hispanics in a manner more consistent with the self-reports. Similarly, the plausibility of the random recruitment assumption can be affected if the times during which interviews are conducted exclude some subjects, if the incentives are not salient for some groups of respondents, and if the remoteness of the interview site limits access. More generally, the random recruitment assumption is only plausible if members of the target population have reasonably easy and comfortable access to the interview site along with appropriate incentives to take advantage of that access.

Testing the random recruitment assumption using comparisons of network composition as revealed by self-reports and by recruitment behavior has an inherent limitation. Comparisons are possible for visible personal characteristics, such as gender, race/ethnicity, or homelessness. However, they are not possible for terms that are not public knowledge, such as HIV status or frequency of HIV risk behavior. If the random recruitment assumption is satisfied with respect to issues of public knowledge, the assumption of random recruitment is made more plausible for other issues.

This section reports on a comparison based on gender of both waves of the SATH-CAP study. This characteristic was selected because with rare exceptions, it is an unambiguous matter of public knowledge. In contrast, a term such as race/ ethnicity is increasingly ambiguous for respondents of diverse racial/ethnic background. Furthermore, whereas gender is a characteristic that is meaningful at all four sites, race/ethnicity would not be meaningful in St. Petersburg, Russia where race/ethnicity is homogeneous. Analysis was limited to recruitment of drug users, a group that encompassed most respondents at all sites, because non-drug-using MSM and MSMW are by definition male. Therefore, non-drug users, and respondents whose drug use status was unknown, were eliminated from the analysis.

The central issue for the comparison is how much bias might have been introduced into the RDS population estimate by violations of the assumption. To measure this potential bias, a two-step procedure was employed. First, the population estimate was calculated in the usual manner for an RDS analysis. This involved calculating the population estimate based on two terms, proportional cross-group recruitment (e.g., the proportion of males who recruit females) and the estimated network size for each group. This yields the RDS population estimate.

The second step is to calculate cross-group recruitment based on network selfreports. This reveals what actual cross-group recruitment would have been had respondents recruited in a manner consistent with their self-reports. For example, respondents were asked how many male drug users that they knew and how many female drug users that they knew. The self-report-based cross-group recruitment could then be calculated. For example, for male respondents, cross-gender recruitment of drug users is the number of female drug users who are known divided by the sum of the number of male and female drug users who are known. This self- 
report-based cross-group recruitment proportion was then entered into the RDS estimator equation to calculate what the population estimate would have been for this group had actual recruitment patterns exactly coincided with the self-reports.

The difference between the RDS population estimator and the self-report-based population estimator indicates the potential bias resulting from violations of the random recruitment assumption. Figure 1 shows the outcome of these analyses where, for each of the four study sites, the bar with the diagonal lines is the RDS estimate, the bar with the dots is the self-report estimate, and the error bars correspond to $95 \%$ confidence intervals.

The estimates for the Raleigh-Durham and St. Petersburg sites are convergent, with each estimate lying within the other's confidence intervals. This correspondence provides support for the random recruitment assumption. In contrast, the Chicago site estimates are more divergent, though the confidence intervals are adjacent, so the difference is not statistically significant.

Finally, the Los Angeles estimates are divergent, with no overlap in the confidence intervals, so the difference is statistically significant. An examination of the origins of this discrepancy reveals that it lies principally in the recruitment patterns of males. Though they reported knowing $24.2 \%$ females, they in fact recruited only $6 \%$. Consequently, they appear to have massively under-recruited females. The pattern for recruitment by females reflects a smaller divergence between actual recruitment and the self-report, but a similar pattern. Females reported knowing $65.2 \%$ males, but recruited only $54.5 \%$. Consequently, using self-reports as a baseline, each gender under-recruited members of the opposite gender.

No definitive explanation of the Los Angeles site's results is possible because neither self-reports nor recruitment behavior constitutes a gold standard for measuring social network composition. As a result, when discrepancies arise, it is not clear which term is at fault. Alternative arguments are possible. On the one hand, comparisons of the accuracy of self-report network indicators have found reports of network size to be among the more reliable and valid. ${ }^{23}$ On the other hand, behavioral indicators such as actual recruitment behavior are frequently viewed as more reliable and valid than self-report-based data. The validity of selfreports can be assessed, at least in part, by examining internal consistency and

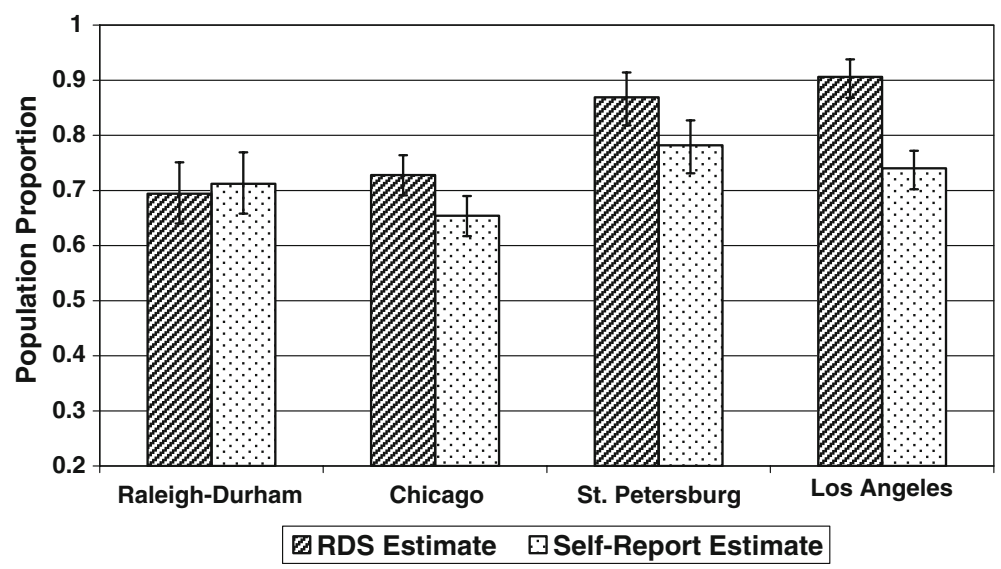

FIGURE 1. Analysis of random recruitment by gender. 
plausibility of the reports. In the Los Angeles data set, internal consistency was imperfect; $74.3 \%$ of respondents (710/956) reported knowing a number of drug users that did not equal the numbers of male and female drug users that were known, (e.g., one respondent reported knowing 70 drug users, of whom three were male and three were female). Also, some self-reports appeared implausible, (e.g., $31.7 \%$ of drug users (303/956) reported not knowing any other drug users, and $2 \%$ of respondents (19/956) failed to answer the question). Overall, $82.8 \%$ of respondents' self-reports (792/956) were in some respect problematic. Unfortunately, no comparable method for assessing the internal consistency of recruitment patterns is available; however, an indirect measure is available for assessing the relative role of problematic self-reports versus recruitment patterns in producing discrepant estimates. If the discrepancy between the two types of estimates results from errors in self-reports, a positive relationship would be expected between the percentage of problematic self-reports and the discrepancy between the estimates, and the two types of estimates would become convergent when problematic selfreports are rare. Evidence for this pattern emerges from a cross-site comparison of problematic self-reports. In the site where the estimates are most divergent, problematic self-reports are more common, $82.8 \%$, than in the sites where estimates are more convergent, $9.4 \%, 10 \%$, and $17.2 \%$, for Chicago, Raleigh-Durham, and St. Petersburg, respectively. This pattern suggests that errors in self-reports may be contributors to the discrepancies.

Several explanations of the discrepancy that reflect either discrepancies between network composition and recruitment behavior or errors in the self-reports are possible. First, the discrepancy could reflect greater social cohesion within as compared to across genders, so members of each gender are more effective at recruiting from their own group, thereby inducing a discrepancy between recruitment and network composition. Should this prove to be the case, then RDS population estimates could potentially be improved if the network size question is reframed to focus only on close associates and exclude distant acquaintances. However, it is not clear why this pattern appeared at sites other than LA for males, but not for females. Another possibility is that cross-gender associations are differentially valued, so self-reports could have been distorted by a social acceptability bias in which members of each gender exaggerate their number of crossgender peers. Should the social desirability explanation prove to be the case, the validity of network self-reports for assessing the random recruitment assumption could be refined based on measures of social desirability, and this might differentiate the LA case from the other cases.

In sum, evidence consistent with the random recruitment assumption was found at two sites (Raleigh-Durham and St. Petersburg), and discrepant evidence was found at the Los Angeles site. However, interpretation of the discrepancy given that the origin of the disparity-either errors in self-reports or divergence between recruitment behavior and network composition-remains unclear. Clearly, more research is needed both on recruitment behavior in RDS and on how respondents formulate their descriptions of network composition. Such research might lead to ways to structure the recruitment relationship more effectively to ensure that the random recruitment assumption is satisfied and more valid and reliable means for eliciting self-reports of network composition. A useful place to begin would be cases where the discrepancy is especially large, e.g., a closer study both of recruitment by gender among male respondents in LA and of the process by which this group reported its network composition. 
Sample Equilibrium Sample equilibrium in RDS is the point in the recruitment process at which the sample stabilizes, i.e., the sample becomes independent of the choice of initial seeds and, theoretically, reflects the composition of the population being studied. When we examined race/ethnicity for the US sites, we found that all three of them eventually reached equilibrium, although they did so at different points in the recruitment process (see Figure $2 \mathrm{a}-\mathrm{c}$, which reflects equilibrium for phase 1 of the study).

Waves are the number of levels in a recruitment chain that follow from an initial seed. The Raleigh-Durham sample stabilized almost from the start in both phases of the study seed (Figure 2a), indicating that proportions recruited during the first waves of the study accurately reflected the reported sample composition for race/ ethnicity. The Los Angeles sample (Figure 2b) reached equilibrium for race/ethnicity by about wave 10 in phase 1 and by about wave 6 during phase 2 (data not shown). The Chicago sample reached equilibrium in about wave 6 at its largest interview site (Figure 2c). We present Chicago data from only one interview site as an illustration.

\section{DISCUSSION}

\section{Efficient Recruitment of DU and MSM}

The strengths of the dual higher risk group RDS approach to sampling are apparent. Because RDS facilitates the recruitment of large numbers of participants in a short time, it was possible to interview more than 3,000 participants in slightly more than 1 year across our four sites and more than 8,000 total across both phases of the study. Efficiency in RDS generally refers to a group's efficiency with respect to peer recruitment. It is defined here as the ratio of the group's number of recruits to the number of the group who recruited them. ${ }^{36}$ There was a high ratio of recruits to seeds at all of the US sites, but not at the St. Petersburg sites. Ratios were as follows: Raleigh-Durham, 1889:96; Los Angeles, 1522:120; Chicago, 3760:47; and St. Petersburg, 765:156.

However, although recruitment was efficient at most sites, the number of seeds required appears higher than that reported in other RDS studies, particularly for phase 2 of our study. This need for a greater number of seeds in phase 2 likely resulted from our prohibiting phase 1 subjects to enroll in phase 2; that is, participants in phase 1 probably included a substantial proportion of the most easily enrolled persons: those who resided or spent time near the study sites, were part of local street scenes, and were particularly amenable to joining the study. In consequence, many of these people likely were not available when phase 2 began. Recruiting for phase 2 thus faced a challenge that was absent in phase 1 . Moreover, by limiting the number of coupons in the early stages of phase 2 , we may have inadvertently caused chains to "die out" earlier than they did in phase 1 .

\section{Identification of Overlapping At-Risk Populations}

One of the distinctive features of this study was its use of RDS to simultaneously recruit two overlapping risk populations thought to be at high risk for HIV infection: MSM and DU. Because RDS recruitment occurs peer to peer, recruitment links may be used to examine the overlap between MSM and DU networks. A person's personal network is defined for RDS purposes as those who are acquaintances, friends, or those closer than friends who qualify for inclusion in the study and have been seen or otherwise contacted within a specified interval such 
a

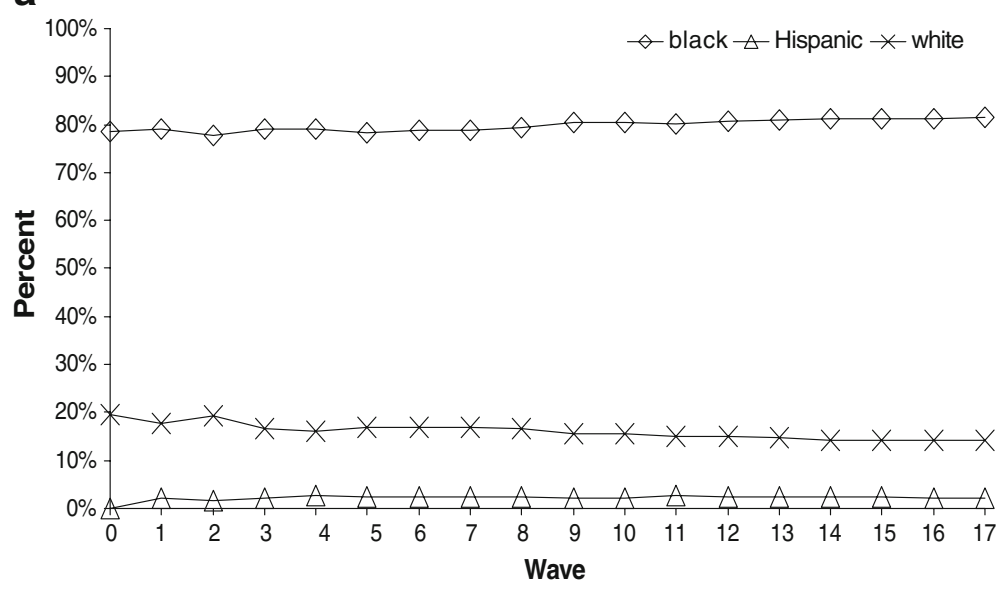

b
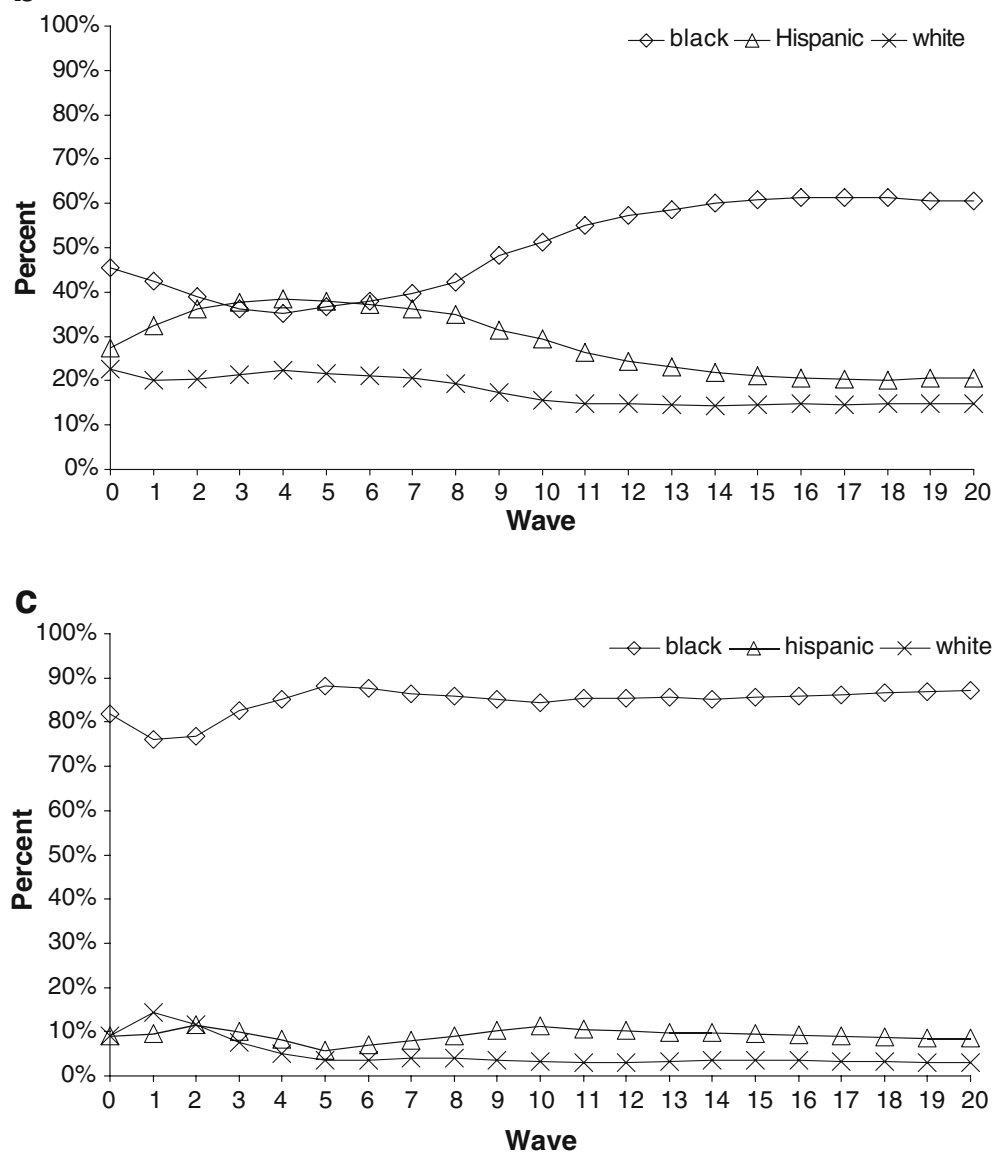

FIGURE 2. Race/ethnicity proportions by wave (phase 1): a Raleigh-Durham, b Los Angeles, c Chicago. 
as 6 months. Equivalently but more simply, a person's personal network is the set of persons that the respondent knows whom he or she could legitimately recruit into the study. ${ }^{36}$ For example, in Russia, homophily was very high among both MSM and DU recruits, with MSM demonstrating an almost complete preference for recruiting other MSM. When out-group recruitment occurred, it almost always involved MSM/DU and rarely included heterosexual DU. In Russia, a very small MSM population $(n=22)$ appeared to serve as a bridge between the two higher risk and non-overlapping populations.

The pattern observed in the US sites was very different. DU and MSM demonstrated a much lower in-group recruitment tendency. In all three US cities, DU and MSM showed a moderate bias against recruiting one another, with DU also tending not to recruit MSM/DU. This tendency, however, was much weaker than the one observed in our Russian site. It should also be noted that a great majority of men reporting as MSM in our US sites were MSM/DU, making this group much less a bridge than a directly overlapping risk group.

\section{Identification of a Large Population of MSMW}

One of the more interesting results from the recruitment strategy was the enrollment of a large population of men who reported having sex with both men and women in the previous 6 months. The large proportions of men reporting bisexual behavior is consistent with recent suggestions in the literature of high rates of bisexual behavior among men recruited as MSM ${ }^{56-61}$ and also among men not recruited as such. ${ }^{62,63}$ These studies suggest that as many as $27 \%$ of MSM may also be having sex with women. ${ }^{56}$ Our study reinforces these findings.

In St. Petersburg, $19.2 \%$ of the 180 MSM, both DU and non-DU, reported having sex with a woman in the 6 months prior to the interview. This fraction was even higher in the USA where MSMW were a substantial proportion not just of MSM but of all men recruited into the study. These proportions of MSMW among all men recruited into the study ranged from $17 \%$ (in Chicago) to $20 \%$ (in RaleighDurham) to $35 \%$ (in Los Angeles). Most of the MSMW at the US sites were non-gay identified, lower income, crack cocaine users, and black. Clearly, this group has potential as a bridge for HIV transmission between MSM and their female sexual partners.

\section{Study Limitations: Recruitment Bias, Violations of RDS Assumptions, and Generalizability}

As stated earlier, one of the basic theoretical underpinnings of RDS is that respondents recruit randomly from their personal networks. Our data indicate that this assumption likely was violated for gender at our Los Angeles site and suggest that the assumption also could have been violated for other characteristics, such as race/ethnicity. These findings limit statements of the sample's representativeness with respect to point prevalence estimates between groups. In addition, significant differences between the percentage of Hispanics who refused coupons and the percentage of Hispanics recruited suggest possible non-participation bias for Hispanics in Los Angeles. We have also considered the impact of these problems on assumptions underlying RDS such as those related to Markov models (e.g., random sampling without "memory"), ${ }^{25}$ but our conclusion was that minor violations of those assumptions meant that equilibrium would be reached more slowly; thus, no further adjustments were required. 
It is clear that the RDS sampling approach did not result in a diverse sample of MSM and DU across age and income groups at any of our US sites, limiting the extent to which we were able to generalize our results beyond the lower socioeconomic networks from which our participants appear to be drawn. In the USA, our MSM, DU, and MSM/DU samples were all older, primarily black or Hispanic, and very low income. Conspicuously absent from the RDS recruited sample in the US were younger, white, and middle- and upper-income DU and MSM. Attempts to recruit higher income MSM and DU were unsuccessful; recruitment chains either ended quickly or dropped back into lower income participants. In St. Petersburg, Russia, participants appeared to be predominantly heroin-using IDU and similarly very low income (with approximately $49 \%$ reporting they were unemployed), but they were much younger than their US counterparts.

There are a number of reasons why individuals from higher socioeconomic status (SES) groups might not have been recruited to the study in the USA. First, participants were required to come to established interview sites that were generally located in lower income and minority neighborhoods. Second, burdens associated with participation were relatively high, including an interview process that took over an hour to complete and a requirement to provide biological samples for testing. In all sites, this involved a blood draw, and in Los Angeles, male participants were required to also provide an anal swab for human papilloma virus testing. Third, the study relied entirely on money or gifts as incentives for participation and did not attempt to encourage participation for any other purpose, such as health promotion or scientific value. Promoting participation for socially redeeming purposes and teaming with community organizations to encourage participation might have resulted in the recruitment of individuals with higher SES. Indeed, survey researchers have concluded that the decision to participate in research involves the interaction of a number of factors, such as context in which survey takes place, behavior of person asking for survey participation, sponsorship and topic of survey, monetary or other kinds of incentives, amount and type of respondent burden, and degree of "match" between interviewer and respondent. ${ }^{64}$ These factors may well play out differently for higher and lower income subjects and for MSM and DU.

\section{CONCLUSIONS}

In summary, RDS was an efficient means for recruiting a large number of lower income DU and MSM, but generally was not successful at recruiting from all eligible segments, such as groups with higher incomes. The lack of reach across SES levels in the USA may indicate a balkanization of groups, but it may also reflect a general failure on our part to make the study relevant and attractive to individuals who require more than monetary incentives to participate. It may also be the case that since RDS requires an acknowledged and reciprocal social tie in the determination of eligible recruits, anonymous ties are left as potential means for disease transmission between groups that might not appear to intersect socially. Future studies will need to examine this possibility, with special attention to venues where SES groups might anonymously intersect.

Use of RDS in the simultaneous recruitment of DU and MSM resulted in the identification of a population of DU MSMW. This group requires additional study because it clearly holds potential for bridging populations at varied risk for HIV and other infectious diseases. 
Finally, although the sample may not represent the entire population of our dually recruited risk groups, the recruited samples at each of our sites are certainly large enough to warrant attention on their own, and it does not seem unreasonable to view them as representative of lower income DU and MSM residing proximal to our interview sites. Despite uncertainty about the representativeness of the samples at each site, the participants may still be of particular importance to policymakers. The poor, unemployed, marginally housed, and uninsured DU and MSM in this study represent a group exhibiting a range of behaviors that place them at high risk from both acquiring and transmitting HIV. The group is also extremely vulnerable to arrest and incarceration, heavily dependent on publicly funded services, and likely to require publicly funded treatments for substance abuse, HIV, and other infectious diseases.

\section{ACKNOWLEDGMENT}

Support for this grant was provided by NIDA grants U01DA017377; U01DA017378; U01DA017394; U01DA017387; and U01DA017373.

OPEN ACCESS This article is distributed under the terms of the Creative Commons Attribution Noncommercial License which permits any noncommercial use, distribution, and reproduction in any medium, provided the original author(s) and source are credited.

\section{REFERENCES}

1. Whiteside A, Barnette T. AIDS in the Twenty-First Century, 2nd ed. New York: Palgrave Macmillan; 2006.

2. Booth RE, Lehman WE, Kwiatkowski CF, Brewster JT, Sinitsyna L, Dvoryak S. Stimulant injectors in Ukraine: the next wave of the epidemic? AIDS Behav. 2008; 12: 652-661.

3. de Azevedo RC, Botega NJ, Guimaraes LA. Crack users, sexual behavior and risk of HIV infection. Rev Bras Psiquiatr. 2007; 29(1): 26-30.

4. Drumright LN, Patterson TL, Strathdee SA. Club drugs as causal risk factors for HIV acquisition among men who have sex with men: a review. Subst Use Misuse. 2006; 41 (10-12): 1551-1601. doi:10.1080/10826080600847894.

5. Schwarcz S, Scheer S, McFarland W, et al. Prevalence of HIV infection and predictors of high-transmission sexual risk behaviors among men who have sex with men. Am J Public Health. 2007; 97(6): 1067-1075. doi:10.2105/AJPH.2005.072249.

6. Zule WA, Costenbader E, Coomes CM, et al. Stimulant use and sexual risk behaviors for HIV in rural North Carolina. J Rural Health. 2007; 23(Suppl): 73-78. doi:10.1111/ j.1748-0361.2007.00127.x.

7. Hall HI, Song R, Rhodes P, et al. Estimation of HIV incidence in the United States. JAMA. 2008; 300(5): 520-529. doi:10.1001/jama.300.5.520.

8. CDC. HIV/AIDS surveillance report, 2005. vol 17. Revised edition. Atlanta: US Department of Health and Human Services; 2007.

9. Neal JJ, Fleming PL, Green TA, Ward JW. Trends in heterosexually acquired AIDS in the United States, 1988 through 1995. J Acquir Immune Defic Syndr Hum Retrovirol. 1997; 14(5): 465-474.

10. CDC. Trends in HIV/AIDS diagnoses among men who have sex with men-33 States, 2001-2006. JAMA. 2008; 300(5): 497-499.

11. AIDS Foundation East-West. Officially registered HIV cases by region of the Russian Federation-1 January 1987 through 30 June 2007. Moscow. Accessed on: March 16, 2009. Available at: http://www.afew.org/index.php?id=33. 
12. UNAIDS/WHO. Report on the global HIV/AIDS epidemic, June 1998. Geneva: UNAIDS and WHO; 2006.

13. Shoptaw S, Reback CJ. Methamphetamine use and infectious disease-related behaviors in men who have sex with men: implications for interventions. Addiction. 2007; 102(Suppl 1): 130-135. doi:10.1111/j.1360-0443.2006.01775.x.

14. Colfax G, Coates TJ, Husnik MJ, et al. Longitudinal patterns of methamphetamine, popper (amyl nitrite), and cocaine use and high-risk sexual behavior among a cohort of San Francisco men who have sex with men. J Urban Health. 2005; 82(1 Suppl 1): i62i70. doi:10.1093/jurban/jti025.

15. Plankey MW, Ostrow DG, Stall R, et al. The relationship between methamphetamine and popper use and risk of HIV seroconversion in the multicenter AIDS cohort study. J Acquir Immune Defic Syndr. 2007; 45(1): 85-92. doi:10.1097/QAI.0b013e3180417c99.

16. Wang MQ, Collins CB, Kohler CL, DiClemente RJ, Wingood G. Drug use and HIV risk-related sex behaviors: a street outreach study of black adults. South Med J. 2000; 93(2): 186-190.

17. Wingood GM, DiClemente RJ. The influence of psychosocial factors, alcohol, drug use on African-American women's high-risk sexual behavior. Am J Prev Med. 1998; 15(1): 54-59. doi:10.1016/S0749-3797(98)00027-0.

18. Somlai AM, Kelly JA, McAuliffe TL, Ksobiech K, Hackl KL. Predictors of HIV sexual risk behaviors in a community sample of injection drug-using men and women. AIDS Behav. 2003; 7(4): 383-393. doi:10.1023/B:AIBE.0000004730.62934.ed.

19. Timpson SC, Williams ML, Bowen AM, Keel KB. Condom use behaviors in HIV-infected African American crack cocaine users. Subst Abus. 2003; 24(4): 211-220. doi:10.1023/ A:1026043529583.

20. Hwang LY, Ross MW, Zack C, Bull L, Rickman K, Holleman M. Prevalence of sexually transmitted infections and associated risk factors among populations of drug abusers. Clin Infect Dis. 2000; 31(4): 920-926. doi:10.1086/318131.

21. Abdala N, Krasnoselskikh TV, Durante AJ, Timofeeva MY, Verevochkin SV, Kozlov AP. Sexually transmitted infections, sexual risk behaviors and the risk of heterosexual spread of HIV among and beyond IDUs in St. Petersburg, Russia. Eur Addict Res. 2008; 14(1): 19-25. doi:10.1159/000110407.

22. Abdul-Quader AS, Heckathorn DD, McKnight C, et al. Effectiveness of respondentdriven sampling for recruiting drug users in New York City: findings from a pilot study. $J$ Urban Health. 2006; 83(3): 459-476. doi:10.1007/s11524-006-9052-7.

23. Heckathorn D, Semaan S, Broadhead R, Hughes J. Extensions of respondent-driven sampling: a new approach to the study of injection drug users aged 18-25. AIDS Behav. 2002; 6(1): 55-67. doi:10.1023/A:1014528612685.

24. Magnani R, Sabin K, Saidel T, Heckathorn D. Review of sampling hard-to-reach and hidden populations for HIV surveillance. AIDS. 2005; 19(Suppl 2): S67-S72. doi:10.1097/01.aids.0000172879.20628.e1.

25. Heckathorn D. Respondent driven sampling: a new approach to the study of hidden samples. Soc Probl. 1997; 44(2): 174-199.

26. Frost SD, Brouwer KC, Firestone Cruz MA, et al. Respondent-driven sampling of injection drug users in two U.S.-Mexico border cities: recruitment dynamics and impact on estimates of HIV and syphilis prevalence. J Urban Health. 2006; 83(Suppl 6): i83-i97. doi:10.1007/s11524-006-9104-z.

27. Stormer A, Tun W, Guli L, et al. An analysis of respondent driven sampling with injection drug users (IDU) in Albania and the Russian Federation. J Urban Health. 2006; 83(Suppl 6): i73-i82. doi:10.1007/s11524-006-9105-y.

28. Wang J, Carlson R, Falck R, Siegal H, Rahmen A, Li L. Respondent-driven sampling to recruit MDMA users: a methodological assessment. Drug Alcohol Depend. 2005; 78: 147-157. doi:10.1016/j.drugalcdep.2004.10.011.

29. Wang J, Falck RS, Li L, Rahman A, Carlson RG. Respondent-driven sampling in the recruitment of illicit stimulant drug users in a rural setting: Findings and technical issues. Addict Behav. 2007; 32(5): 924-937. doi:10.1016/j.addbeh.2006.06.031. 
30. Ramirez-Valles J, Heckathorn DD, Vazquez R, Diaz RM, Campbell RT. From networks to populations: the development and application of respondent-driven sampling among IDUs and Latino gay men. AIDS Behav. 2005; 9(4): 387-402. doi:10.1007/s10461-0059012-3.

31. Johnston LG, Khanam R, Reza M, et al. The Effectiveness of respondent driven sampling for recruiting males who have sex with males in Dhaka, Bangladesh. AIDS Behav. 2008; 12(2): 294-304. doi:10.1007/s10461-007-9300-1.

32. Millett GA, Ding H, Lauby J, et al. Circumcision status and HIV infection among black and Latino men who have sex with men in 3 US cities. J Acquir Immune Defic Syndr. 2007; 46(5): 643-650. doi:10.1097/QAI.0b013e31815b834d.

33. Mimiaga MJ, Goldhammer H, Belanoff C, Tetu AM, Mayer KH. Men who have sex with men: perceptions about sexual risk, HIV and sexually transmitted disease testing, and provider communication. Sex Transm Dis. 2007; 34(2): 113-119. doi:10.1097/01.olq. 0000225327.13214.bf.

34. Deiss RG, Brouwer KC, Loza O, et al. High-risk sexual and drug using behaviors among male injection drug users who have sex with men in 2 Mexico-US border cities. Sex Transm Dis. 2008; 35(3): 243-249.

35. Iguchi MY, Bux DA, Lidz V, Kushner H, French JF, Platt JJ. Interpreting HIV seroprevalence data from a street-based outreach program. J Acquir Immune Defic Syndr. 1994; 7: 491-499.

36. Heckathorn D. Extensions of respondent-driven sampling: analyzing continuous variables and controlling for differential recruitment. Sociol Methodol. 2007; 37: 151-207. doi:10.1111/j.1467-9531.2007.00188.x.

37. Robinson WT, Risser JM, McGoy S, et al. Recruiting injection drug users: a three-site comparison of results and experiences with respondent-driven and targeted sampling procedures. J Urban Health. 2006; 83(Suppl 6): i29-i38. doi:10.1007/s11524-006-9100-3.

38. Grau LE, Dasgupta N, Phinney Harvey A, et al. Illicit use of opioids: is Oxycontin ${ }^{\circledR}$ a gateway drug? Am J Addict. 2007; 16: 166-173. doi:10.1080/10550490701375293.

39. Heimer R. Critical issues and further questions about respondent-driven sampling: comment on Ramirez-Valles, et al. (2005). AIDS Behav. 2005; 9(4): 403-408. doi:10.1007/s10461005-9030-1. discussion 409-413.

40. Brewer DD, Garrett SB, Kulasingam S. Forgetting as a cause of incomplete reporting of sexual and drug injection partners. Sex Transm Dis. 1999; 26(3): 166-176. doi:10.1097/ 00007435-199903000-00008.

41. Brewer DD, Webster CM. Forgetting of friends and its effects on measuring friendship networks. Soc Networks. 1999; 21: 361-373. doi:10.1016/S0378-8733(99)00018-0.

42. Bell DC, Belli-McQueen B, Haider A. Partner naming and forgetting: recall of network members. Soc Networks. 2007; 29(2): 279-299. doi:10.1016/j.socnet.2006.12.004.

43. Scott G. "They got their program, and I got mine": a cautionary tale concerning the ethical implications of using respondent-driven sampling to study injection drug users. Int J Drug Policy. 2008; 19(1): 42-51. doi:10.1016/j.drugpo.2007.11.014.

44. Ouellet LJ. Cautionary comments on an ethnographic tale gone wrong. Int J Drug Policy. 2008; 19: 238-240.

45. Broadhead RS. Notes on a cautionary (tall) tale about respondent-driven sampling: a critique of Scott's ethnography. Int J Drug Policy. 2008; 19: 235-237.

46. Prachand NG. Clarifying the ethnographer's role in Chicago's HIV behavioural surveillance-injecting drug users cycle, 2005: response to Scott. Int J Drug Policy. 2008; 19: 244-245.

47. Friedman S, Bolyard M, Khan M, et al. Group sex events and HIV/STI in an urban network. J Acquir Immune Defic Syndr. 2008; 49(4): 440-446. doi:10.1097/QAI.0b013e3181893f31.

48. Rothenberg R, Muth SQ, Malone S, Potterat JJ, Woodhouse DE. Social and geographic distance in HIV risk. Sex Transm Dis. 2005; 32(8): 506-512. doi:10.1097/01.olq. 0000161191.12026.ca. 
49. Needle R, Fisher D, Weatherby N, et al. Reliability of self reported HIV risk behaviors of drug users. Psychol Addict Behav. 1995; 9: 242-250. doi:10.1037/0893-164X.9.4.242.

50. NIDA. National Institute on Drug Abuse Risk Behavior Assessment. In: Abuse NIoD, ed. Rockville; 1991.

51. Mansergh G. Findings from the first large multi-city U.S. sample of high-risk substance abusing men who have sex with men: baseline results from the Project Mix behavioral intervention trial. Paper presented at: 16th International AIDS Conference; Toronto.

52. Chesney MA, Koblin BA, Barresi PJ, et al. An individually tailored intervention for HIV prevention: baseline data from the EXPLORE Study. Am J Public Health. 2003; 93(6): 933-938. doi:10.2105/AJPH.93.6.933.

53. CDC. HIV/STD risks in young men who have sex with men who do not disclose their sexual orientation-six U.S. Cities, 1994-2000. MMWR Morb Mortal Wkly Rep. Accessed on: November 16, 2008. Available at: http://www.cdc.gov/mmwr/preview/ mmwrhtml/mm5205a2.htm.

54. Gorbach PM, Drumright LN, Daar ES, Little SJ. Transmission behaviors of recently HIVinfected men who have sex with men. J Acquir Immune Defic Syndr. 2006; 42(1): 80-85.

55. Heckathorn D. Assumptions of RDS: analytic vs. functional assumptions. Paper presented at: CDC Consultation on the Analysis of Data Collected Through Respondent-Driven Sampling, 2008; Atlanta, GA.

56. Wheeler DP, Lauby JL, Liu K, Van Sluytman LG, Murrill CS. A comparative analysis of sexual risk characteristics of black men who have sex with men or with men and women. Arch Sex Behav. 2008; 37: 607-707.

57. Wolitski RJ, Jones KT, Wasserman JL, Smith JC. Self-identification as "down low" among men who have sex with men (MSM) from 12 US cities. AIDS Behav. 2006; 10: 519-529. doi:10.1007/s10461-006-9095-5.

58. MacKellar DA, Valleroy LA, Secura GM, et al. Unrecognized HIV infection, risk behaviors, and perceptions of risk among young men who have sex with men: opportunities for advancing HIV prevention in the third decade of HIV/AIDS. J Acquir Immune Defic Syndr. 2005; 38(5): 603-614. doi:10.1097/01.qai.0000141481.48348.7e.

59. Kral AH, Lorvick J, Ciccarone D, et al. HIV prevalence and risk behaviors among men who have sex with men and inject drugs in San Francisco. J Urban Health. 2005; 82(1 Supp.1): i43-i50. doi:10.1093/jurban/jti023.

60. Choi K, Gibson DR, Han L, Guo Y. High levels of unprotected sex with men and women among men who have sex with men: a potential bridge of HIV transmission in Beijing, China. AIDS Educ Prev. 2004; 16(1): 19-30. doi:10.1521/aeap.16.1.19.27721.

61. Montgomery JP, Mokotoff ED, Gentry AC, Blair JM. The extent of bisexual behaviour in HIV infected men and implications for transmission to their female sex partners. AIDS Care. 2003; 15(6): 929-837. doi:10.1080/09540120310001618676.

62. Wohl AR, Johnson DF, Lu S, et al. HIV risk behaviors among African American men in Los Angeles County who self-identify as heterosexual. J Acquir Immune Defic Syndr. 2002; 31: 354-360.

63. Hightow LB, Leone PA, Macdonald PD, McCoy SI, Sampson LA, Kaplan AH. Men who have sex with men and women: a unique risk group for HIV transmission on North Carolina college campuses. Sex Transm Dis. 2006; 33(10): 585-593. doi:10.1097/01. olq.0000216031.93089.68.

64. Groves R, Fowler F, Couper M, Lepkowski J, Singer E, Tourangeau R. Survey Methodology. Hoboken: Wiley; 2004. 